\title{
Propiedades osteoinductivas de la dentina en regeneración ósea. Estudio preliminar
} Osteoinductive properties of the dentin

H. López Sacristán*, M. del Canto Pingarrón**, M. A. Alobera Gracia***, C. Clemente de Arriba****, J. A. Seco Calvo*****

\section{RESUMEN}

Introducción: El concepto de preservación alveolar está relacionado con los procedimientos llevados a cabo tras realizar una exodoncia dentaria con el fin de reducir la reabsorción dentro del alvéolo. La dentina ha sido propuesta como un biomaterial para este fin. Este artículo tiene como propósito evaluar la efectividad de la dentina en la preservación alveolar y contrastar sus propiedades.

Material y Método: Se presentan como parte de un estudio en desarrollo los primeros casos tras realizar exodoncias múltiples. Determinamos un alveolo de estudio preservado con dentina y un alveolo control en el que estabilizamos el coagulo. La estabilidad dimensional de ambos alveolos se valoró mediante CBCT pasadas 8 y 16 semanas. Tras este periodo colocamos dos implantes experimentales de superficie Bioecth®. A las 16 semanas fueron explantados con trefina y sustituidos por implantes convencionales. Estas muestras fueron analizadas histológica e histomorfométricamente.

Resultados: En el análisis tomográfico apreciamos mayor estabilidad dimensional en el lado estudio. Histológicamente observamos ausencia de células inflamatorias, o signos de reacción a cuerpo extraño, además de partículas de dentina completamente rodeadas de hueso neoformado, y frentes osteogénicos partiendo de partículas de dentina.

Discusión: Los resultados obtenidos a partir de este estudio en humanos están en consonancia con lo que ha sido descrito en la literatura. Observamos a nivel tomográfico menor variación dimensional en los alveolos donde se llevó a cabo la preservación con dentina particulada, estos resultados sumados al análisis histológico contrastan las propiedades descritas para injertos particulados de dentina no desmineralizada, biocompatibilidad, osteoconducción y osteoinducción.

PALABRAS CLAVE: "Dentina”, "interfase hueso-implante”, “oseointegración”, "regeneración ósea”, "material biocompatible", "trasplante", "aloinjerto", "injerto de hueso alveolar".

\section{ABSTRACT}

Introduction: The concept of alveolar preservation is related to the procedures carried out after performing a tooth extraction in order to reduce resorption within the alveolus. Dentin has been proposed as a

\footnotetext{
* $\quad$ Profesor del Máster en Cirugía Bucal, Implantología y Periodoncia por la Universidad de León. C/ Alfonso IX 24004, León. España.

* * Director del Máster en Cirugía Bucal, Implantología y Periodoncia por la Universidad de León. C/ Alfonso IX 24004, León. España.

*** Director del Máster en Cirugía Bucal, Implantología y Periodoncia por la Universidad de León. C/ Alfonso IX 2 4004, León. España.

**** Profesora Titular Universidad de Alcalá. Departamento de ciencias morfológicas y cirugía, escuela de Medicina, Universidad de Alcalá. Alcalá de Henares 28871, Madrid. España

***** Profesor Titular Universidad de León. Departamento de Enfermería y Fisioterapia, Campus de Vegazana, 24071, León. España.
} 
biomaterial for this purpose. The aim of this article is to evaluate the effectiveness of dentin in alveolar preservation and to contrast its properties.

Material and Method: The first cases are presented as part of a work in progress study after multiple extractions. It is determined a study socket preserved with dentin and a control socket in which the clot is stabilized.The dimensional stability of both sockets was assessed by CBCT after 8 and 16 weeks. After this period, two experimental Bioecth ${ }^{\circledR}$ surface implants were placed. After 16 weeks these were explanted with trephine and replaced with conventional implants. The samples were analyzed histologically and histomorphometrically.

Results: In the tomographic analysis it was observed greater dimensional stability on the study side. It was noted the absence of inflammatory cells, or signs of foreign body reaction in the histology. In addition, dentin particles were completely surrounded by newly formed bone, and appeared osteogenic fronts associated with these particles.

Discusion: The results obtained from this study in humans are in line with what has been described in scientific papers. At the tomographic level, it was observed less dimensional variation in sockets where the preservation with particulate dentin was carried out. These results added to the histological análisis, contrast the properties described for particulate grafts of non-demineralized dentin, biocompatibility, osteoconduction and osteoinduction

KEY WORDS: "Dentin", "Bone-Implant Interface", "Osseointegration”, "Bone Regeneration", "Biocompatible Materials", "Transplantation", "Allografts", "Alveolar Bone Grafting".

Fecha de recepción: 19 de octubre de 2020

Fecha de aceptación: 26 de octubre de 2020

H. López Sacristán, M. Del Canto Pingarrón, M. A. Alobera Gracia, C. Clemente de Arriba, J. A. Seco Calvo. Propiedades osteoinductivas de la dentina en regeneración ósea. Estudio preliminar. 2021; 37, (1): 39-46

\section{INTRODUCCIÓN}

El tejido óseo es un tejido dinámico que se encuentra en un equilibrio constante a través de los mecanismos fisiológicos de reabsorción y neoformación óseas.

En ambos huesos maxilares, tanto estudios en animales de experimentación como los realizados en humanos, se ha documentado que la cresta alveolar sufre una reabsorción sustancial después de la extracción de un diente ${ }^{(1,2)}$.

Tras la extracción dental, se establece un coágulo de sangre en el alvéolo que estimula la neoformación ósea dentro de las paredes de éste. Sin embargo, el proceso de curación nunca da como resultado el mantenimiento completo del volumen óseo alveolar original puesto que se produce una remodelación fisiológica ${ }^{(3)}$. Los estudios clínicos, radiológicos e histológicos han indicado que tras la extracción se produce la reabsorción de la pared ósea bucal o externa, el "bundle bound" dependiente del periodonto, y la remodelación de las paredes del alveolo original con diversos grados de cambios dimensionales tanto en altura como en anchura de la cresta alveolar ${ }^{(1,2)}$. La reducción ósea en sentido horizontal es de aproximadamente $1 / 3$ durante las primeras 8 semanas y del $50 \%$ durante el primer año ${ }^{(4,5)}$; mientras que es de 0,8 $\mathrm{mm}$. en sentido vertical durante los 3 primeros meses $^{(1)}$.

Con el fin de minimizar la remodelación tras una extracción dental, se han desarrollado diferentes técnicas que buscan preservar el alveolo. Su objetivo es mantener la mayor parte de la integridad del alveolo mediante la utilización de diferentes sustitutos óseos que aporten soporte mecánico adecuado. Además, estos biomateriales tienen que cumplir las características de biocompatibilidad, osteoconductividad, biodegradabilidad y reemplazo por el propio hueso del paciente ${ }^{(6,8)}$. Este reemplazo se ha puesto en duda en investigaciones recientes, puesto que se ha comprobado que existe una reabsorción lenta, e incluso el mantenimiento de las partículas de biomaterial puede ser beneficioso para el mantenimiento a largo plazo del alveolo ${ }^{(9,10)}$.

En la actualidad la búsqueda de un material de injerto óseo ideal ha llevado a muchos investigadores a con- 
siderar el tejido dental como un posible material de interés para la regeneración ósea. La dentina supone el $85 \%$ de la estructura dental, y podría ofrecer un recurso de fácil obtención con un contenido mineral superior al de cualquier material derivado del hueso (12). La dentina es comparable al hueso autólogo en al menos dos aspectos, osteocompatibilidad y osteoconducción, aportando así una matriz para la neoformación ósea ${ }^{(13,14,15)}$. Recientes estudios sugieren que es portadora de proteínas, comunes con las del hueso y el cemento radicular. Éstas podrían inducir la calcificación y neoformación óseas, siendo su composición similar a la del tejido óseo, derivando ambas de células de la cresta neural, y compuestas por el mismo colágeno tipo $\mathrm{I}^{(14)}$.

La dentina contiene BMPs, que inducen la formación de tejido óseo, y proteínas no colágenas tales como la osteocalcina, osteonectina y fosfoproteína, implicadas en la calcificación del hueso. Su contenido inorgánico es del 70-75\%, mientras que el contenido orgánico es del $20 \%$, aproximadamente. Al menos un $90 \%$ del contenido orgánico de la dentina es colágeno tipo $\mathrm{I}^{(14)}$. Los resultados sugieren que la dentina podría desempeñar un papel fundamental en la reparación temprana del hueso y proporcionar una superficie favorable para la actuación de osteoblastos ${ }^{(15)}$.

En 2003 se informa del primer caso de trasplante de dentina autóloga por el equipo de investigación de $\mathrm{Mu}$ rata. En 2011, Murata M. et al presentan a la dentina como un nuevo biomaterial y también como matriz portadora de BMPs (BMP-2), involucrada en la formación ósea. Realizan un estudio en conejos en el que confirmaron que la matriz de dentina completamente desmineralizada (DDM) induce la formación de hueso en 4 semanas, mientras que la dentina no desmineralizada induce la formación ósea a las 8-12 semanas después de su implantación. Este retraso puede estar producido por la inhibición de la BMP- liberadora por los cristales de apatita. Los tejidos altamente calcificados como la dentina no producen una osteoinducción temprana con formación de hueso esponjoso. Después de la desmineralización de la dentina, siguen quedando tipos bioactivos de BMPs, en especial las BMP-2, BMP-4 y BMP-7, que se unen en matrices ricas de colágeno, al igual que en el hueso ${ }^{\circ}$.

Kim y cols. indican una excelente cicatrización utilizando autoinjertos de origen dentario, tanto en la evaluación clínica como radiológica e histológica con mantenimiento de la cresta ósea a 31 meses de seguimiento ${ }^{(17)}$.
Por otro lado, del Canto y cols ${ }^{(19)}$ demostraron el mantenimiento dimensional y el incremento densitrométrico de alveolos postextracción preservados con dentina autóloga.

El presente artículo pretende comprobar la capacidad osteinductora de la dentina mediante un análisis tomográfico, histológico e histomorfométrico en humanos, y su influencia en las variaciones del BIC y la densidad de área en implantes experimentales con una superficie ampliamente probada en la actualidad.

\section{MATERIAL Y MÉTODO:}

El estudio clínico se está llevando a cabo en las instalaciones del Máster en Cirugía Bucal, Implantología y Periodoncia de la Universidad de León. Se seleccionarán para el estudio 30 pacientes, y en este artículo se muestran los resultados preliminares de los primeros 4 casos completados. Antes de la entrar a formar parte del estudio a todos los sujetos se les pide que firmen un formulario de consentimiento informado para demostrar que entienden el alcance del mismo (incluyendo intervenciones quirúrgicas y los riesgos potenciales involucrados), permitiendo la oportunidad de hacer preguntas relacionadas con el estudio y dando información sobre tratamientos alternativos. Esta investigación ha sido avalada por el Comité Ético de la Universidad de León ya que cumple con los criterios estrictos planteados por dicho comité (anexo 1), y así mismo cumple con los acuerdos de la declaración de Helsinki.

\section{CRITERIOS DE INCLUSIÓN:}

- Sujetos de edad igual o mayor a 18 años, de ambos sexos y de cualquier raza.

-Pacientes parcial o totalmente desdentados a los que se indica tratamiento implantológico en el maxilar o la mandíbula.

-Que sean sujetos identificados como candidatos para la colocación de implantes dentales.

Que comprendan que están involucrados en el estudio, incluida la necesidad de visitas de seguimiento. - Con $6 \mathrm{~mm}$. o más de anchura ósea crestal, que será suficiente para colocar un implante cónico con superficie Bioetch@ de $2 \mathrm{~mm}$. de diámetro y $8 \mathrm{~mm}$. de longitud. Tras 16 semanas se realizará explantación para el estudio, seguida de la colocación de un implante más ancho como tratamiento para la reposición prostodóncica necesaria. 
CRITERIOS DE EXCLUSIÓN:

-Adicciones diversas (Tabaquismo, alcoholismo, drogadicción, etc).

-Embarazo.

- Historia de enfermedad sistémica no controlada o de enfermedades crónicas.

-Trastorno de la coagulación o toma de anticoagulantes.

- Tratamiento con bifosfonatos.

- Sujetos con compromiso severo del sistema inmunológico.

- Historial de inestabilidad mental que obstaculice la participación en el estudio.

\section{Diseño DEL ESTUDIO:}

Una vez seleccionado el paciente se procederá a la exodoncia de dientes que no puedan ser mantenidos por causas estéticas, funcionales o rehabilitadoras, previa sindesmotomía de los tejidos blandos. Se seleccionan dos alveolos de condiciones estructurales semejantes que presenten integridad de todas las paredes. El primero de los alveolos queda integrado dentro del grupo de estudio, en el que se realizará una preservación alveolar con dentina y membrana de colágeno. La dentina se obtiene de los dientes extraídos, que previamente ha sido particulada yprocesadasiguiendo los protocolos del sistema Smart Dentin Grinder de Kometabio ${ }$. En nuestro estudio retiramos por completo la corona y, por tanto, cualquier parte de esmalte, y solo utilizamos la raíz de dichos dientes. El segundo alveolo pasa a formar parte del grupo control en el que se estabilizará un coagulo con una membrana de colágeno. Se realiza un control tomográfico de los alveolos en el momento de la preservación, a las 8 y a las 16 semanas, que nos servirá para determinar los cambios volumétricos en uno y otro lecho a nivel radiográfico. Para ello realizaremos mediciones en sentido vertical siguiendo el eje axial del diente, y en sentido horizontal perpendiculares al eje axial (Fig.1).

La segunda parte del estudio comenzará a las 16 semanas de realizadas las preservaciones alveolares, colocando los implantes experimentales que se describen en el próximo apartado. El objetivo será el análisis de las muestras que se obtendrán 4 meses después de la colocación de los implantes, para estudio histológico e histomorfométrico para obtener el BIC (Bone Implant Contact) y la Densidad de Área.

Material implantológico: Se utilizan implantes cónicos de $2 \mathrm{~mm}$. de diámetro en zona crestal y más estre-

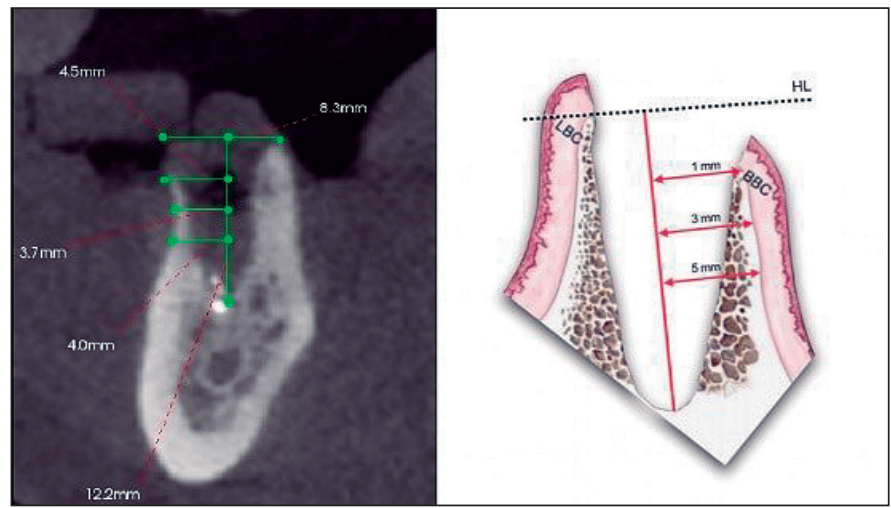

Figura 1. Análisis tomográfico de los cambios dimensionales del alveolo, HL línea horizontal medición de anchura, VL línea vertical eje axial del diente, $\mathrm{BBC}$ cortical ósea vestibular, $\mathrm{LBC}$ cortical ósea lingual. Mediciones: HL, VL, HL a BBC, VL a BBC $1 \mathrm{~mm}$.

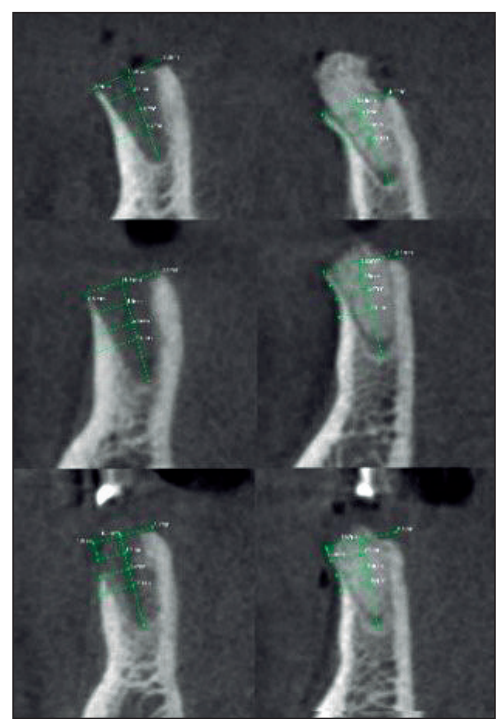

Figura 2. Análisis de la variación dimensional del caso 4 a nivel tomográfico. La columna de la izquierda muestra el alveolo control, y la de la derecha el alveolo donde se realizó la preservación con dentina. Fila 1: postoperatorio, Fila 2: 8 semanas, Fila 3: 16 semanas. cho a modo de guía en apical, y $8 \mathrm{~mm}$ de longitud. Presenta un cuerpo roscado con un paso de 0,25 $\mathrm{mm}$. y con superficie de sustracción Bioetch®. El lecho implantario se lleva a cabo con una primera fresa de lanza y una segunda fresa de 1,8 mm. El implante entra en el lecho guiado por un destornillador universal de la marca Bioner(R).

Tras 16 semanas, se realiza la explantación de los implantes del estudio con una fresa trefina de diámetro interno $4 \mathrm{~mm}$. y externo de $4,5 \mathrm{~mm}$. que, en la mayoría de los casos, asegura la recuperación del implante, con el tejido circundante a su alrededor, en toda su longitud.

A continuación, se introduce la fresa trefina, con el material implantario y tisular en su interior, en formaldehido tamponado al $10 \%$ para su traslado al laboratorio.

Al paciente se le colocará un implante de al menos 5 $\mathrm{mm}$ de diámetro, para rehabilitar la zona donde se ha recogido la muestra. 
Metodología histológica:

La muestra recogida será tratada y estudiada en formol tamponado y luego se enviará al laboratorio de Histología del Departamento de Cirugía, Ciencias Médicas y Sociales de la Universidad de Alcalá. El procesamiento de cada muestra se realizará de la siguiente manera:

1. Inclusión de la muestra en polímeros plásticos.

2. Preparaciones histológicas con cortes de hueso sin decalcificar y con el implante incluido.

3. Corte de las muestras con el sistema EXACT. Estudio histológico de los cortes con la descripción y la diferenciación del tejido óseo en cada muestra.

4. Tinción de las secciones con azul de toluidina, hematoxilina-eosina y/o tricrómico de Masson.

5. Evaluación de la presencia o ausencia de reacción inflamatoria o reacción de cuerpo extraño.

6. Estudio histomorfométrico de la muestra en función de la morfología de la superficie del implante. La muestra se evaluará con el programa MIP-45 evaluando el porcentaje de osteointegración (BIC), la Densidad de Área Ósea alrededor cada implante.

7. Estudio estadístico para evaluar diferencias entre los dos tipos de muestras con test de análisis de la varianza (ANOVA). Este último paso no se ha realizado para este artículo ya que la muestra presentada es insuficiente para llevar a cabo el estudio estadístico.

Las muestras pueden ser cortadas con el sistema EXAKT de dos formas:

1. De 4 a 6 cortes transversales al eje longitudinal de la muestra. Con este sistema se obtienen muchos más datos y disminuye la desviación estándar al realizar la estadística del BIC.

2. Un corte longitudinal al eje de la muestra con lo cual se obtienen menos datos del estudio.

\section{RESULTADOS}

Como ha comentado anteriormente, los resultados que se muestran a continuación son parte de un estudio en desarrollo del cual, en este momento de la investigación, solo se han obtenido datos de cuatro

\begin{tabular}{|c|c|c|c|c|c|c|}
\hline \multicolumn{7}{|c|}{ Tabla1 } \\
\hline CAS01 & $\mathrm{HL}$ & HL-BBC & VL & VL-BBC1 & VL-BBC2 & VL-BBC3 \\
\hline CONTROL POSTOP & 7,6 & 1,3 & 13,1 & 4,3 & 3,4 & 2,9 \\
\hline CONTROL 8 SEMANAS & 5,5 & 0,9 & 11,9 & 3,3 & 3,1 & 2,3 \\
\hline CONTROL 16 SEMANAS & 5,2 & 0,8 & 10,7 & 3,8 & 2,6 & 2 \\
\hline DENTINA POSTOP & 7,2 & 1,4 & 14,5 & 4,9 & 4 & 3,8 \\
\hline DENTINA 8 SEMANAS & 6,8 & 1,4 & 12,9 & 3,6 & 3,5 & 3,5 \\
\hline DENTINA 16 SEMANAS & 6,7 & 1,2 & 12,7 & 3,5 & 3,4 & 3,4 \\
\hline \multicolumn{7}{|l|}{ CASO 2} \\
\hline CONTROL POSTOP & 8,3 & 2,4 & 11,4 & 4,5 & 3,7 & 4 \\
\hline CONTROL 8 SEMANAS & 7,9 & 2 & 10,6 & 3,5 & 3,1 & 3,1 \\
\hline CONTROL 16 SEMANAS & 7,2 & 1,6 & 9,1 & 3,1 & 2,9 & 2,7 \\
\hline DENTINA POSTOP & 8 & 1,8 & 12,7 & 4,5 & 3,7 & 3,1 \\
\hline DENTINA 8 SEMANAS & 7 & 1,5 & 10,7 & 3,9 & 3,5 & 2,8 \\
\hline DENTINA 16 SEMANAS & 6,8 & 0,7 & 10,7 & 3,4 & 3 & 2,8 \\
\hline \multicolumn{7}{|l|}{ CASO 3} \\
\hline CONTROL POSTOP & 7,7 & 4,8 & 12,5 & 4,5 & 4,8 & 5 \\
\hline CONTROL 8 SEMANAS & 7,4 & 4,5 & 12 & 3,4 & 4,5 & 4,9 \\
\hline CONTROL 16 SEMANAS & 6,8 & 4,3 & 11,5 & 2,1 & 4,1 & 4,8 \\
\hline DENTINA POSTOP & 8,6 & 5,3 & 12,6 & 4,8 & 4,7 & 4,5 \\
\hline DENTINA 8 SEMANAS & 8,5 & 4,8 & 11,5 & 4,3 & 4,5 & 4,3 \\
\hline DENTINA 16 SEMANAS & 8,3 & 4,5 & 10,9 & 3,8 & 4,3 & 4,3 \\
\hline \multicolumn{7}{|l|}{ CASO 4} \\
\hline CONTROL POSTOP & 9,2 & 0,9 & 11,2 & 4,1 & 4,8 & 4,9 \\
\hline CONTROL 8 SEMANAS & 8,5 & 0,8 & 11 & 3,8 & 4,3 & 4,6 \\
\hline CONTROL 16 SEMANAS & 7,1 & 0,6 & 10,7 & 3,2 & 3,4 & 4 \\
\hline DENTINA POSTOP & 8,4 & 0,9 & 10,4 & 4,1 & 3,8 & 3,9 \\
\hline DENTINA 8 SEMANAS & 8,3 & 0,6 & 10,3 & 3,9 & 3,6 & 3,7 \\
\hline DENTINA 16 SEMANAS & 8,3 & 0,6 & 10,2 & 3,9 & 3,5 & 3,6 \\
\hline
\end{tabular}

Se recogen los valores en milímetros de la variación dimensional tomográfica en los cuatro pacientes con el estudio completo.

pacientes. Los sujetos de la investigación son tres hombres y una mujer. Todos ellos comprendieron el objetivo de la investigación y aceptaron voluntariamente la participación en el estudio.

\section{RESULTADOS RADIOGRÁFICOS:}

Los resultados que se muestran a continuación en las tablas 1 a 4 son los obtenidos del analísis del cambio dimensional en los alveolos grupo control y grupo dentina para los que se realizó un CBCT inmediatamente posterior a la preservación, a las 8 semanas y a las 16 semanas. Se muestra un ejemplo del análisis que se está llevando a cabo. No se han realizado análisis estadísticos de los casos al considerarse una muestra pequeña y formar parte de un estudio en desarrollo, pero se observan en todos los casos una evidente disminución de la pérdida dimensional, tanto vertical como horizontal en el grupo preservado con dentina. (Fig.2) (Tabla1, Tabla2).

\section{RESULTADOS HISTOLÓGICOS:}

Las muestras se incluyeron en metacrilato para la realización de cortes transversales, en los ca- 


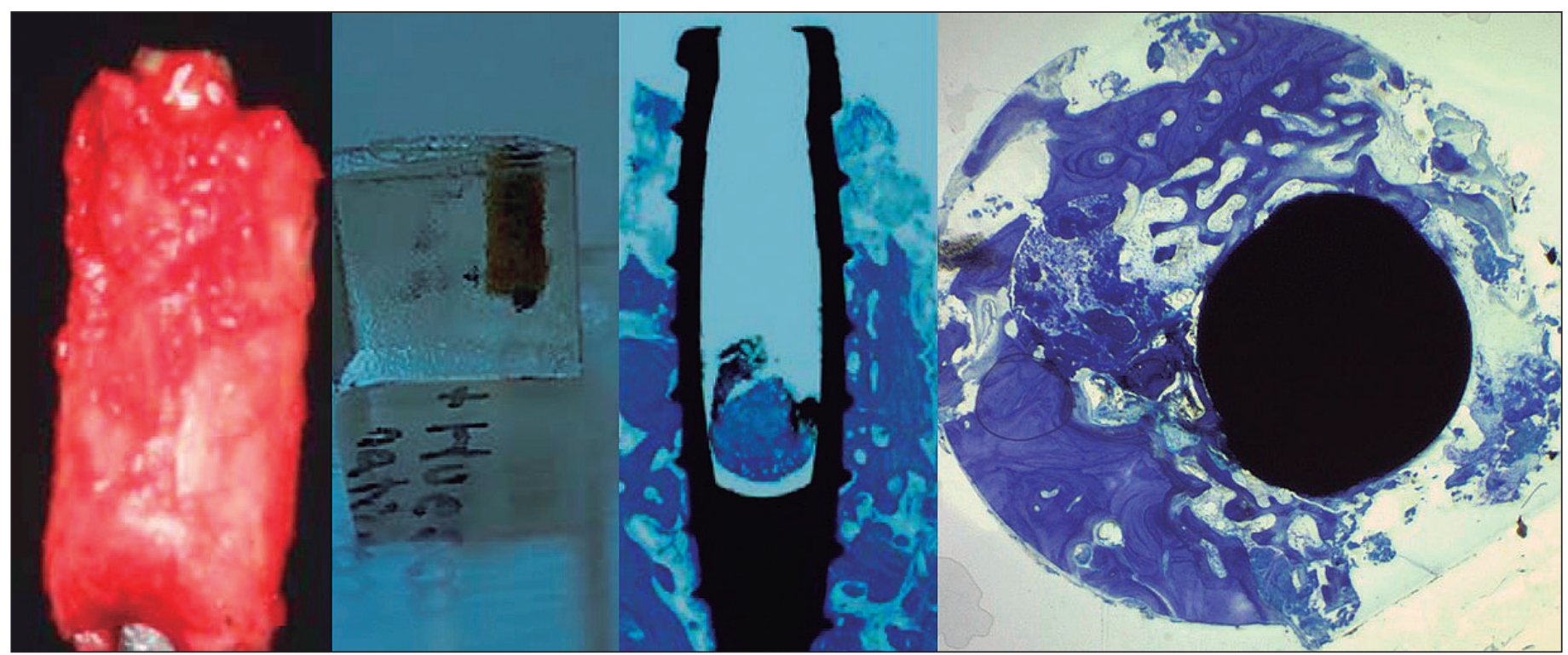

Figura 3. Se observa de izquierda a derecha, muestra recién obtenida, muestra incluida en metacrilato, corte longitudinal de implante con tinción de azul de toluidina y corte transversal con implante tinción de azul de toluidina.

sos 1 y 4 , mientras que las muestras de los casos 2 y 3 fueron cortadas de forma longitudinal con el fin de analizar el contacto hueso implante en toda la superficie del implante (Fig.3).

La histología mostró la existencia de tejido óseo neoformado, tanto en el lado tratado como en el lado control.

Muestras control: No se aprecian células de cuerpo extraño ni inflamatorias a su alrededor. No se produce ninguna modificación de la respuesta ósea normal. En la muestra se aprecian algunas zonas de hueso neoformado en contacto con el implante y en regiones cercanas al mismo.

Muestras con dentina: En el estudio histológico se identifican partículas de dentina, algunas aisladas y otras rodeadas totalmente por hueso neoformado. Independientemente de la presencia de las partículas de dentina, se reconocen algunas zonas de osteointegración con hueso nuevo en contacto con el implante.

En las imágenes histológicas se puede apreciar partículas de dentina completamente rodeadas por hueso sin signos de reacción a cuerpo extraño.

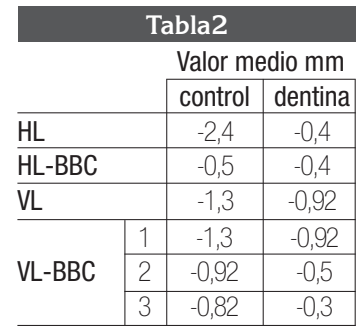

Se recogen los valores medios en milímetros de la variación dimensional de los cuatro pacientes tratados. Se observa una menor pérdida ósea en los casos de preservación alveolar con dentina tanto en sentido vertical (VL y HL-BBC) como en los análisis horizontales (HL y VL-BBC).

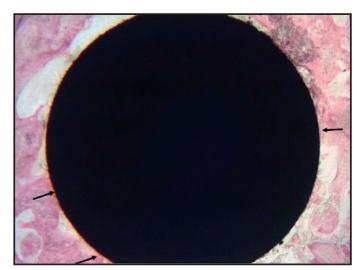

Figura 4. Corte transversal de la muestra. Lado tratado. Aumentos 2'5x. Tinción con hematoxilina eosina. El hueso aparece en color rosa. Existen múltiples puntos de contacto con el implante (flechas).

\section{RESULTADOS HISTOMORFOMÉTRICOS:}

En el estudio se determinan mediante histomorfometría los valores de bone implant contact (BIC) y Densidad de área (Aa) analizándo los cortes histológicos.

Para ello se utiliza el software MIP45 que permite analizar las imágenes transferidas a ordenador desde microscopio o lupa estereoscópica.

No se muestran resultados de medición del BIC y Aa en este momento de la investigación debido a que la muestra es demasiado pequeña. (Fig.4)

\section{DISCUSIÓN}

El propósito de este artículo es demostrar las propiedades de biocompatibilidad, osteoconducción y osteoinducción de la dentina como material de injerto óseo, en el estudio que actualmente está en desarrollo.

La composición de la dentina es similar a la del tejido óseo tanto a nivel mineral como orgánico ${ }^{(12,14,15,18)}$, siendo este último en su mayoría colágeno tipo I y proteínas que podrían estimular la calcificación ósea en diferentes momentos de la cicatrización del alveolo. En la literatura ya se ha 
descrito la regeneración con este material de forma particulada ${ }^{(12)}$ posteriormente a la extracción dental, lo cual nos aporta un material con características únicas de fácil obtención y manipulación.

Tal y como se ha diseñado el estudio, nos permite analizar el comportamiento del injerto con respecto a un grupo control, en diferentes estadíos de la cicatrización. Este grupo de investigación publicó en 2018 un estudio en el que se concluía que en los alveolos que se realizaba una preservación alveolar con dentina la contracción era menor que ante un grupo control ${ }^{(19)}$. Pese a que con la muestra actual presentada no se ha llevado a cabo un análisis estadístico de los datos, se aprecia en las tablas 1, 2, 3 y 4 que los niveles de contracción tanto en sentido horizontal (HL) como en sentido vertical (VL) son menores en el lado donde se realizó la preservación con dentina.

Pasadas 16 semanas de la preservación, realizamos la reentrada con el fin de colocar los implantes experimentales de superficie Bioecth®. En este momento podemos observar la cicatrización de los alveolos, tal y como describen autores como Cardaropoli D. et al o Chaitanya Pradeep J. et al ${ }^{(20,21)}$, visualmente la cicatrización del alveolo donde se realizó la preservación con dentina es completamente igual a la de cualquier lecho donde no se realizó ningún injerto (Fig.5).

En consonancia con los hallazgos manifestados tanto en modelo animal como humano por otros autores como Murata $M$. et al, Cardaropoli D. et al, y Calvo JL. et al ${ }^{(16,20,22)}$, el análisis histológico de la muestra nos permite ver el comportamiento de las partículas de dentina en relación con el medio. Se observó que no se produce ninguna reacción inflamatoria, ni de reacción a cuerpo extraño, por el contrario, se pueden apreciar diferentes partículas rodeadas de hueso neoformado, constatando las propiedades de biocompatibilidad y osteoconducción de la dentina particulada. Además, encontramos neoformación ósea a partir de estas partículas con frentes de osteoblastos que muestran la capacidad de la dentina de estimular la diferenciación de células óseas, por tanto, se reafirma histológicamente su capacidad osteoinductiva descrita por autores como Hussain I. et al, Kim Y. et al, y Murata $M^{\cdot} \cdot(13,14,16)$ (Fig.6).

En este momento de la investigación los resultados obtenidos del cálculo de porcentaje de BIC y Aa son escasos como para poder valorarlos estadísticamente, pero parece observarse que la presencia de dentina podría aumentar la densidad de área a las 16 se-

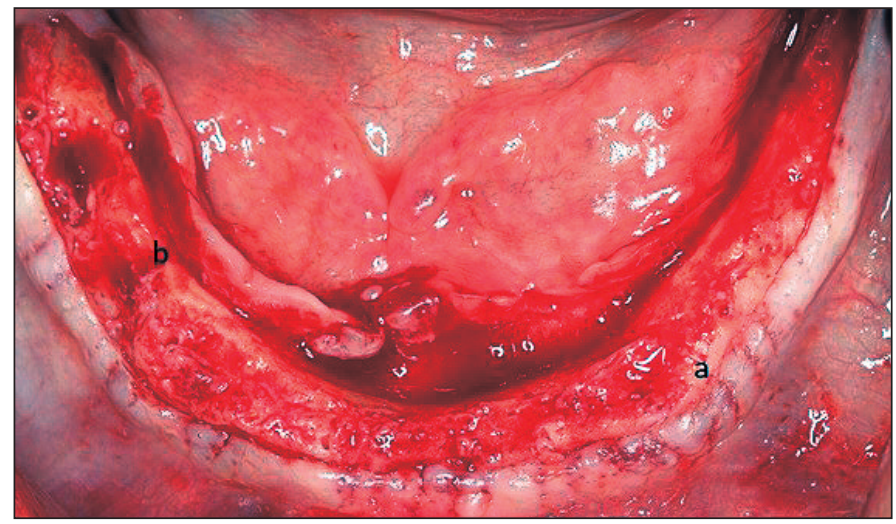

Figura 5. Foto de reentrada a las 16 semanas caso 2, a: lado estudio (dentina), b: lado control.

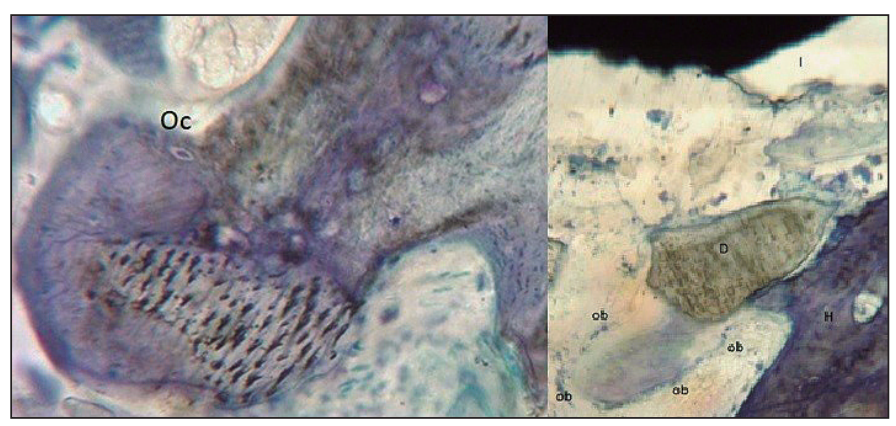

Figura 6. Izquierda: Detalle de partícula de dentina rodeada por hueso neoformado. La dentina se ve como un fragmento surcado por trazos negros paralelos entre sí. El huso parece de color azul-morado, con alguna laguna osteocítica (Oc) en su interior. Aumentos 10x. Derecha: Detalle a 6x aumentos de dentina. La partícula de dentina tiene hueso neoformdo a su derecha y en la parte inferior hay hueso en neoformacion, con presencia de un frente de osteoblastos. (ob). I: implante experimental de superficie Bioetch ${ }^{\circledR}$, D: partícula de dentina, $\mathrm{H}$ : hueso.

manas de colocación con respecto al grupo control. No obstante, son datos preliminares que no permiten sacar conclusiones definitivas, por lo que es necesario ampliar la muestra.

\section{CONCLUSIONES}

- Los resultados obtenidos son parte de un estudio en desarrollo, por lo que es recomendable ampliar la muestra con el fin de contrastar estadísticamente las conclusiones que se detallan a continuación.

-El análisis tomográfico muestra que la preservación alveolar con dentina particulada disminuye los cambios dimensionales del alveolo a las 16 semanas.

- Los resultados preliminares del estudio a nivel histológico avalan las propiedades de biocompatibilidad, 
osteoconducción y osteoinducción de la dentina. Por lo tanto, la dentina puede considerarse como una alternativa prometedora a la utilización de hueso autólogo como injerto.

- Los resultados a nivel histomorfométrico que pretenden determinar el BIC y la densidad de área (Aa) de forma comparativa no son concluyentes en este momento de la investigación, siendo necesario ampliar la muestra para obtener resultados estadísticos.

\section{AGRADECIMIENTOS}

Este estudio se está llevando a cabo gracias a la colaboración de Bioner Sistemas Implantológicos@ que facilita todo el material implantológico detallado en el apartado material y métodos. Queremos agradecer la estrecha colaboración mostrada con el Máster de Cirugía Bucal, Implantología y Periodoncia por la Universidad de León por parte de esta empresa y de su equipo humano.

\section{REFERENCIAS BIBLIOGRÁFICAS}

1. Araujo MG, Lindhe J. Dimensional ridge alterations following tooth extraction. An experimental study in the dog. J Clin Periodontol 2005; 32: 212-8.

2. Schropp L, Wenzel A, Kostopoulos L, Karring T. Bone healing and soft tissue contour changes following single-tooth extraction: a clinical and radiographic 12-month prospective study. Int J Periodontics Restorative Dent 2003; 23: 313-23.

3. Vignoletti F, Abrahamsson I. Quality of reporting of experimental research in implant dentistry. Critical aspects in design, outcome assessment and model validation. J Clin Periodontol 2012; 9: 6-27.

4. Carlsson GE, Bergman B, Hedegard B. Changes in contour of the maxillary alveolar process under immediate dentures. A longitudinal clinical and x-ray cephalometric study covering 5 years. Acta Odontol Scand 1967; 25: 45-75.

5. Seibert JS. Reconstruction of deformed, partially edentulous ridges, using full thickness onlay grafts. Part II. Prosthetic/periodontal interrelationships. Compend Contin Educ Dent 1983; 4: 549-62.

6. Jensen SS, Aaboe M, Pinholt EM, Hjorting-Hansen E, Melsen F, Ruyter IE (1996) Tissue reaction and material characteristics of four bone substitutes. Int J Oral Maxillofac Implants 11: 55-66.

7. Fugazzotto PA. GBR using bovine bone matrix and resorbable and non resorbable membranes. Part 1: histologic results. Int J Periodontics Restorative Dent 2003; 23: 361-9.

8. Fugazzotto PA. GBR using bovine bone matrix and resorbable and nonresorbable membranes. Part 2: Clinical results. Int J Periodontics Restorative Dent 2003; 23: 599-605.

9. Jensen SS, Broggini N, Hjorting-Hansen E, Schenk R, Buser D. Bone healing and graft resorption of autograft, anorganic bovine bone and beta-tricalcium phosphate. A histologic and histomorphometric study in the mandibles of minipigs. Clin Oral Implants Res 2006; 17: 237-43.

10. Jensen SS, Bornstein MM, Dard M, Bosshardt DD, Buser D. Comparative study of biphasic calcium phosphates with different HA/TCP ratios in mandibular bone defects. A longterm histomorphometric study in minipigs. J Biomed Mater Res B: Appl Biomater 2009; 90:171-81.

11. Ten Heggeler JM, Slot DE, Van der Weijden GA. Effect of socket preservation therapies following tooth extraction in non-molar regions in humans: a systematic review. Clin Oral Implants Res. 2011; 22:779-88.

12. Binderman I, Hallel G, Nardy C, Yaffe A, Sapoznikov L. A Novel Procedure to Process Extracted Teeth for Immediate Grafting of Autogenous Dentin. J Interdiscipl Med Dent Sci. 2014; 2(6): 154.

13. Hussain I, Moharamzadeh K, Brook IM, José de Oliveira Neto, Patrício, Salata LA. Evaluation of osteoconductive and osteogenic potential of a dentin-based bone substitute using a calvarial defect model. Int J Dentistry.2012.

14. Kim Y, Um I, Lee JK, Kim K, Murata M. Healing mechanism and clinical application of autogenous tooth bone graft material.: INTECH Open Access Publisher; 2013.

15. Al-Namnam NM, Shanmuhasuntharam P, Ha KO, Siar CH. Processed Allogenic Dentine as A Scaffold for Bone Healing: An in vivo study. Aust J Basic Applied Sci 2010; 4(12):5932-40.

16. Murata M, Um I, Kim K, Mitsugi M, Akazawa T, Kim Y. Human dentin as novel biomaterial for bone regeneration. INTECH Open Access Publisher;2011.

17. Young-Kyun, Su-Gwan K, Ji-Hyun B, In-Woong U, Ji-Su O, Kyung-In J. Guided Bone Regeneration Using Autogenous Tooth Bone Graft in Implant Therapy: Case Series. Implant Dentistry. 2014; 23(2): 138-43.

18. Linde A. Dentin matrix proteins: composition and possible functions in calcification. Anat Rec. 1989;224:154-66.

19. Canto-Diaz A, Elio-Oliveros J, Canto-Diaz M, Alobera-Gracia MA, Canto-Pingarrón M, Martínez-González JM. Use of autologous tooth-derived graft material in the post-extraction dental socket. Pilot study. Med Oral Patol Oral Cir Bucal. 2019 Jan 1;24(1):53-60.

20. CArdaropoli D., Nevins M., Schupbach P. New bone formation using an extracted tooth as a biomaterial: A case report with histologic evidence. Int J Periodontics Restorative Dent 2019; 39:157-163.

21. Pradeep Joshi C., Bernardo D’Lima C., Chandrashekhar Samat U., Ashok Karde P., Ganpat Patil A., Hemchandra Dani N., Comparative Alveolar Ridge Preservation Using Allogenous Tooth Graft versus Free-dried Bone Allograft: A Randomized, Controlled, Prospective, Clinical Pilot Study.Contemp Clin Dent. 2017 Apr-Jun; 8(2): 211-217.

22. Calvo Guirado JL., Sapoznikov L., Cegarra del Pino P., Delgado Ruíz RA., Fernandez-Dominguez M., Alexandre Gehrke Sérgio. A new procedure for processing extracted teeth for immediate grafting in post-extraction sockets. An experimental study in American Fox Hound dogs. Ann. Anat. 217 (2018) 14-23.

\section{DIRECCIÓN DE CORRESPONDENCIA:}

Avenida de Valladolid 2, Primera Planta Oficina 3. Aldeamayor de San Martín, 47162, Valladolid. España. Tlfn.:665217603. hernan32.hl@gmail.com 